\title{
Love life, scientific anti-cancer, better life
}

Author/Photo: Yi Cheng, from Tongji Cancer Center, Tongji Hospital, Tongji Medical College, Huazhong University of Science and Technology, Wuhan 430030, China

Received: 4 May 2010 / Revised: 7 May 2010 / Accepted: 15 May 2010

(c) Huazhong University of Science and Technology and Springer-Verlag Berlin Heidelberg 2010

The Chinese Anti-cancer Association launched the 16th National Tumor Prophylaxis and Treatment Week in April, 2010. In order to highlight the week's spirit of "Love life, scientific anti-cancer, better life", the Tongji Cancer Center and Thoracic Surgery Department held lectures and advisory services in the outpatient lobby of Cancer Center on April 20th, 2010. The content of lectures involves the prophylaxis, diagnosis and treatment of lung cancer.

The lectures, including the oncological half and the surgical half, were hosted by professor Yuan Chen. In the first half, doctor Qiu Hong, introduced the basic information about lung cancer. She pointed that: "smoking and drinking are the risk factors of lung cancer. More than $80 \%$ of lung cancer is caused, at least partially, by longtime smoking. If the smoking index (the cigarettes consumed one day times the years of smoking) is higher than 400 , the person is likely to suffer lung cancer. As for alcohol, most people said drinking in an appropriate amount is good for health. But what is the appropriate amount?

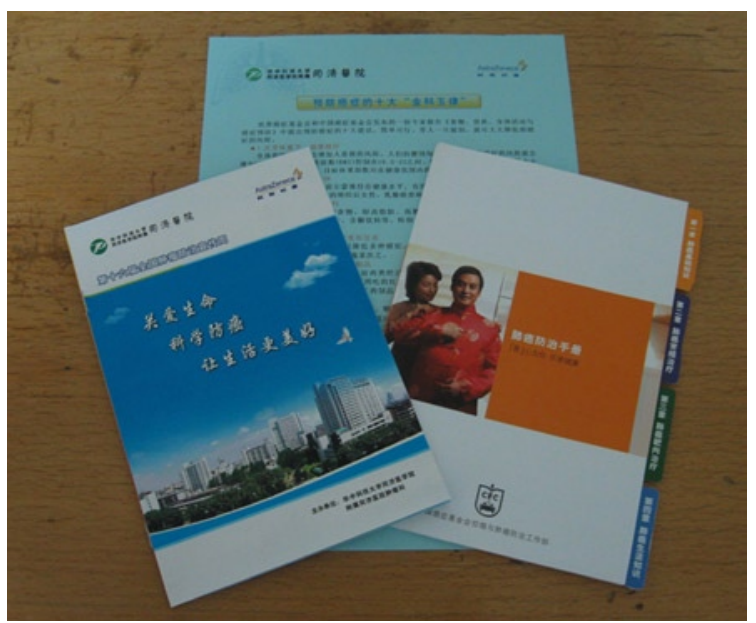

Fig. 2 The leaflets and manuals of the lectures and advisory services

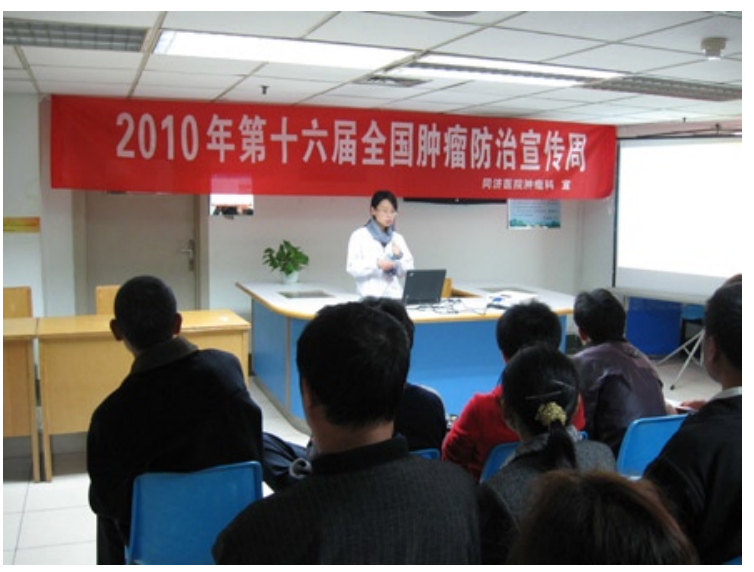

Fig. 1 Doctor Qiu Hong, from Tongji Cancer Center, was introducing the basic information about lung cancer

I suggest $30-50 \mathrm{~mL}$ of white spirit, or $50-100 \mathrm{~mL}$ of red wine, per day."

"Thus, two slogans of the week, 'protect environment, refuse tobacco, far from cancer' and 'control alcohol intake, reduce cancer risk', prove themselves importance."

Then, doctor Li Zhang introduced the chemotherapy, radiotherapy, and molecular targeted therapy towards lung cancer. Comprehensive treatment would be the developing tendency in the near future.

In the second half, doctor Jianing Wang introduced the fundamental information about surgical treatment of lung cancer. She emphasized the importance of early diagnosis and treatment. Doctor Shenling Fu introduced the details of surgery, and the matters needed attention.

After the lectures, the doctors held advisory services. The patients and their relatives participating in the lectures and advisory services, got some leaflets and manuals about lung cancer, provided by AstraZeneca. The patients and their relatives set high values on the lectures and advisory services. They hoped that more activities would be held in future. 